\title{
Abuso digital nas relações amorosas: um estudo das representações sociais com universitários brasileiros
}

\author{
Cyber dating abuse: a study of social representations with Brazilian university \\ students
}

\section{Abuso digital en las relaciones amorosas: un estudio de las representaciones sociales con estudiantes universitarios brasileños}

\author{
Jaqueline Gomes Cavalcanti ${ }^{1}$, ORCID 0000-0002-3068-404X \\ Maria da Penha de Lima Coutinho ${ }^{2}$, ORCID 0000-0003-3961-2402 \\ Adriele Vieira de Lima Pinto ${ }^{3}$, ORCID 0000-0003-4126-1795
}

\author{
${ }^{123}$ Universidade Federal da Paraíba. Brasil
}

\begin{abstract}
Resumo: O presente estudo objetivou apreender as representações sociais (RS) de universitários brasileiros acerca do abuso digital nos relacionamentos amorosos (ADRA). Participaram da pesquisa 447 participantes os quais responderam à Técnica de Associação Livre de Palavras e ao Questionário de Abuso Digital nos Relacionamentos Amorosos (QADRA). Os dados apontaram para uma alta prevalência de envolvimento no ADRA, tanto em vitimização (87,6\% controle/monitoramento; 34,7\%, agressão direta); quanto, em perpetração (92,6\% controle/monitoramento; $25,1 \%$, agressão direta). Além disso, os resultados indicaram que os elementos estruturantes das RS se ancoraram na incerteza relacional contemporânea e na concepção de dominância dentro dos relacionamentos íntimos. Quanto as evocações do núcleo periférico, o ADRA foi ancorado na violação dos direitos à privacidade e nas suas causas e consequências, perpassando aspectos psicoafetivos e sociais. Finalmente, os dados revelaram que o "ciúme" se apresenta como elemento central e organizador das RS desse fenômeno, motivando ações virtuais abusivas.
\end{abstract}

Palavras-chave: abuso digital; parceiros íntimos; relacionamentos amorosos; representações sociais

Abstract: The present study aimed at apprehending the social representations (SR) of Brazilian university students about digital Cyber Dating Abuse (CDA). A total of 447 participants participated in the study, responding to the Free Word Association Technique and the Cyber Dating Abuse Questionnaire (CDAQ). The data pointed to a high prevalence of involvement in CDA, both in victimization (87.6\% control / monitoring, 34.7\%, direct aggression); (92.6\% control/monitoring, $25.1 \%$, direct aggression). In addition, the results indicated that the structuring elements of SR were anchored in contemporary relational uncertainty and the conception of dominance within intimate relationships. Concerning the evocations of the peripheral nucleus, CDA was anchored in the violation of the rights to privacy and its causes and consequences, permeating psycho-affective and social aspects. Finally, the data revealed that "jealousy" presents itself as the central and organizing element of RS of this phenomenon, motivating abusive virtual actions.

Keywords: cyber abuse; intimate partners; love relationships; social representations 
Resumen: El objetivo del presente estudio fue aprehender las representaciones sociales (RS) de universitarios brasileños acerca del abuso online en el noviazgo (AON). Participaron de la encuesta 447 participantes los cuales respondieron a la Técnica de Asociación Libre de Palabras y al Cuestionario Abuso Online en el Noviazgo (CAON). Los datos apuntaron a una alta prevalencia de participación en el ADRA, tanto en victimización (87.6\% control / monitoreo, 34.7\%, agresión directa); como en la perpetración (92.6\% control / monitoreo, $25.1 \%$, agresión directa). Además, los resultados indicaron que los elementos estructurantes de las RS se anclaron en la incertidumbre relacional contemporánea y en la concepción de dominación dentro de las relaciones íntimas. En cuanto a las evocaciones del núcleo periférico, el CAON fue anclado en la violación de los derechos a la privacidad y en sus causas y consecuencias, atravesando aspectos psicoactivos y sociales. Finalmente, los datos revelaron que los "celos" se presentan como elemento central y organizador de las RS de ese fenómeno, motivando acciones virtuales abusivas.

Palabras clave: abuso online; parejas íntimas; relaciones amorosas; representaciones sociales

Como citar:

Cavalcanti, J.G., Coutinho, M.P.L., \& Pinto, A.V.L. (2020). Abuso digital nas relações amorosas: um estudo das representações sociais com universitários brasileiros. Ciencias Psicológicas, 14(2), e-2312. doi: https://doi.org/10.22235/cp.v14i2.2312

Correspondência: Jaqueline Gomes Cavalcanti. Universidade Federal da Paraíba, Brasil. E-mail: gomes.jaqueline@gmail.com. Maria da Penha de Lima Coutinho; e-mail: mplcoutinho@gmail.com. Adriele Vieira de Lima Pint; e-mail:adri.vlp8@gmail.com

A violência entre pessoas com laços íntimos entre si não se trata de um fenômeno recente, haja vista serem encontradas ainda, nas décadas de 60 e 70, pesquisas acerca do abuso familiar, envolvendo maus tratos contra filhos e mulheres. Desde então, verifica-se uma notoriedade das temáticas "violência conjugal"; "violência doméstica" e "violência marital". Não obstante, atualmente, alguns autores tem investigado esses temas sob o ângulo da "violência entre parceiros íntimos" (VPI), por considerá-lo um termo mais abrangente, uma vez que tal abuso não se restringe apenas a espaços domésticos e conjugais, nem ocorre somente do homem para mulher (Conceição, Bolsoni, Lindner \& Coelho, 2018; Neves, 2008).

Nesta direção, novos contextos de VPI são mencionados na literatura, como: violência no namoro (Beserra et al., 2015; Pimentel, Moura, \& Cavalcanti, 2017), violência nas relações homossexuais (Elísio, Neves, \& Paulos, 2018; Santos \& Caridade, 2017; Wu et al., 2015), e bissexuais (Turell, Brown, \& Herrmann, 2018); violência entre parceiros íntimos interraciais (Brownridge, Taillieu, Chan, \& Piotrowski, 2018); VPI dentro do ambiente virtual ou Abuso Digital nos Relacionamentos Amorosos (ADRA) (Burke, Wallen, Val-Smith, \& Knox, 2011; Cutbush, Ashley, Kan Hampton, \& Hall, 2010; Flach \& Deslanches, 2017; Tokunaga, 2011; Zweig, Dank, Yanher, \& Lachman, 2013).

Destarte, o presente estudo focará no ADRA, fenômeno que se caracteriza por ameaças, insultos, humilhações ou comportamentos de controle, usando meios eletrônicos, destinados a provocar angústia no parceiro (Borrajo, Gámez-Guadix, Pereda, \& Calvete, 2015a; Flach \& Deslanches, 2017; Van Ouytsel, Ponnet, \& Walrave, 2016). Se por um lado, o ADRA emerge no contexto da violência entre parceiros íntimos, por outro, se dá pelo uso crescente de tecnologias de informação e comunicação (TIC) por casais. 
Assim sendo, a tecnologia, enquanto um meio que possibilita uma infinidade de relações, para além dos limites geográficos, temporais, culturais, cognitivos, passou a ser ferramenta para perpetradores humilharem, deflagrarem e agredirem seus companheiro(a)s (Deans \& Bhogal, 2019; Flach \& Deslanches, 2017; Peskin et al., 2017).

Neste sentido, o abuso por meio da tecnologia pode ser facilitado pela sensação de anonimato e falta de sinais físicos; pela possibilidade de ocorrência em qualquer lugar ou momento (mesmo após o término do relacionamento); não sendo necessária a presença do agressor (Melander, 2010; Stonard, Bowen, Lawrence, \& Price, 2015), podendo atingir a uma maior audiência de pessoas (Runions, Shapka, Dooley, \& Modecki, 2013; Slonje \& Smith, 2008).

Com isso, verifica-se uma maior desinibição dos comportamentos; e um maior controle do indivíduo sobre as interações dos parceiros podendo tornar o abuso digital tão prevalente quanto o presencial. Acerca disso, em estudos internacionais, são verificados taxas de vitimização que variam de 1,1\% a 91,9\%; e índices de perpetração entre 2,7 \% e 97,7\% (Durán \& Martínez-Pecino, 2015; Reed, Tolman, \& Ward, 2017; Smith et al., 2018; Zweig et al., 2013).

Pesquisas alertam também para implicações severas aos envolvidos neste fenômeno, a saber: distúrbios no sono, sintomas depressivos e ansiosos, ideação suicida, envolvimento em comportamentos delinquentes, e implicações negativas para o rendimento escolar (Flach \& Deslanches, 2017).

Fatores relacionados ao envolvimento no ADRA podem ser diversos, como de ordem individual (sexo e idade); relativos ao relacionamento (histórico de abuso em relacionamentos anteriores); familiares; psicológicos (raiva, ciúme, hostilidade); relacionados às percepções; normas e crenças (Deans \& Bhogal, 2019; Hancock, Keast, \& Ellis, 2017; Peskin et al., 2017; Ramos, Miller, Moss, \& Margolin, 2017; Sánchez, Muñoz-Fernández, \& Ortega-Ruíz, 2015; Van Ouytsel et al., 2016).

Por conseguinte, esse conjunto de fatores elucida para o fato de que o ADRA não pode ser estudado de forma descontextualizada, mas por meio de uma perspectiva psicossocial, considerando as relações sociais que a constitui, bem como os significados atribuídos pelos atores sociais ao fenômeno. Desse, modo, o presente estudo se ancora na Teoria das Representações Sociais (TRS) como base analítica do saber compartilhado pelos atores sociais.

A TRS, cujo principal expoente foi Serge Moscovici, diz respeito a uma abordagem teórica metodológica, inserida na Psicologia Social. Parte da concepção de que as representações sociais (RS) se constituem de um conjunto de conceitos, proposições e explicações originadas na vida cotidiana no curso de comunicações interpessoais (Moscovici, 2012). Pode ser ainda entendida como uma forma de conhecimento, socialmente, elaborada e partilhada, com busca a construção de uma realidade comum a um grupo social (Jodelet, 1989). Neste sentido, seu conceito pode agrupar diversos elementos, como: informativos, ideológicos, normativos, crenças, valores, percepções, opiniões, preconceitos, atitudes (Jodelet, 2001).

Para formação das RS dois processos são necessários, a objetivação e a ancoragem (Moscovici (1981/2012). O primeiro diz respeito a forma como se organizam os elementos constituintes da representação, bem como o percurso pelo qual tais elementos adquirem materialidade; e o segundo corresponde a assimilação do novo conceito por objetos já existentes no sistema cognitivo, transformando o não familiar em familiar (Vala, 2013).

Dentro da TRS destaca-se a abordagem estrutural outorgada por Abric (1998) que desmembra as RS em dois elementos: os centrais, que correspondem a parte estável e consensual da RS cuja função é gerar o significado básico da representação e determinar a organização global de todos os elementos. E os periféricos, que tem um caráter mais flexível, permite o ajustamento a mudanças contextuais e a adaptação à realidade concreta; e protege o sistema central (Abric, 1998).

Sob a ótica desses aportes teóricos, esta pesquisa compreende o abuso digital dentro dos relacionamentos amorosos como um fenômeno inscrito em uma produção histórico social, na qual são os indivíduos que as (re)elaboram, as(re)significam e as (re)interpretam segundo interesses 
sociais e culturais. Desse modo, o ADRA não pode ser entendido fora das relações sociais que a constitui, mas na própria dinâmica do relacionamento e da sociedade que a fundou. Neste sentido, para esta investigação procurou-se apreender as representações sociais de universitários brasileiros acerca do abuso digital nos relacionamentos amorosos.

\section{Método}

\section{Tipo de Investigação}

Tratou-se de uma pesquisa quantitativa, exploratória e descritiva, com corte transversal, e amostra não probabilística por conveniência.

\section{Amostra}

Participaram deste estudo 447 participantes, com idades entre 18 a 56 anos $(M=28,97$; $D P=6,05)$, sendo a maioria mulheres $(79,9 \%)$; heterossexuais $(85,4 \%)$, com escolaridade correspondente a pós-graduação $(54,4 \%)$; e que estavam em um relacionamento atual $(70,5 \%)$. Desses, 55,5\% moravam na região Nordeste do Brasil, 22,8\%, na região Sudeste, 13,6\%, na região Sul, $4 \%$, no região Norte, e $3,4 \%$, na região Centro Oeste. Finalmente, a maior parte da amostra assumiu gastar mais de 6 horas, por dia, na internet $(35,6 \%)$, e no celular $(31,1 \%)$.

\section{Instrumentos}

Para apreender as Representações sociais utilizou-se a Técnica de Associação Livre de Palavras (TALP) que consiste em evocar cinco palavras relacionadas à indutora (abuso digital nos relacionamentos amorosos).

Para mensurar as taxas de envolvimento foi utilizado o Questionário de Abuso Digital nos Relacionamentos Amorosos (Borrajo et al., 2015a) composta por duas escalas, uma de vitimização (10 itens) e outra de perpetração (10 itens), ambas compostas por dois fatores que contabilizam 20 itens: Agressão Direta (AD, 11 itens); e Controle/Monitoramento (C, 9 itens). Tais componentes apresentaram uma consistência interna satisfatória, em sua versão original, através dos índices de Alfa de Cronbach: Agressão Direta (vitimização $\alpha=0.84$; perpetração $\alpha=0,73$ ); e Controle/Monitoramento (vitimização $\alpha=0.87$; perpetração $\alpha=0,81$ ). No contexto brasileiro, tais índices foram corroborados tanto para escala de vitimização (AD $\alpha=$ de 0,71 a 0,78 ; C $\alpha=$ de 0,81 a 0,90) quanto para escala de perpetração ( $\mathrm{AD} \alpha=0,67$ a 0,80; $\mathrm{C} \alpha=$ de 0,84 a 0,86 ) (Cavalcanti, Coutinho, Nascimento \& Pinto, 2020). Essa medida foi respondida tomando por base uma escala de 5 pontos, que varia de $1=$ " nunca " a $6=$ " geralmente, com escores totais que variam de 20 a 120. Valores altos indicam maior frequência de comportamentos de abuso digital, seja como vítima, na escala de vitimização, seja como agressor, na escala de perpetração.

\section{Procedimentos Éticos}

Esta pesquisa foi submetida ao Comitê de Ética em Pesquisa da instituição dos autores (retirado para avaliação cega), em que se seguiu todos os termos éticos citados pelo Conselho Nacional de Saúde, Brasil, conforme as Resoluções 466/12 e 510/2016. Após aprovado (CAAE: 80433517.4.0000.5188), foi apresentado o Termo de Consentimento Livre e Esclarecido (TCLE) para os universitários.

\section{Procedimentos de Coleta de Dados}

Os participantes foram convidados a responderem a um questionário, via formulário eletrônico, pelo recurso do Google Docs. Para isso, foi utilizado o compartilhamento do mesmo em comunidades acadêmicas. Não obstante, antes de iniciar a pesquisa, o respondente era esclarecido quanto ao objetivo do estudo e orientado sobre o caráter voluntário e confidencial. Caso concordasse em responder, o instrumento era disponibilizado ao participante. Convém 
destacar que inicialmente era apresentado ao respondente a TALP e em seguida o Questionário de Abuso Digital nos Relacionamentos Amorosos e o sociodemográfico, para que não houvesse influências desses na TALP. O tempo de duração média para preenchimento foram de 15 minutos.

\section{Procedimentos de Análise de Dados}

Para análise do QADRA e sociodemográfico foram feitas análises descritivas, como média, desvio padrão através do software IBM SPSS 21. Quanto aos dados advindos da TALP, esses foram analisados no software Iramuteq, através da análise prototípica. A análise prototípica busca identificar a estrutura representacional a partir dos critérios de frequência e ordem de evocação das palavras provenientes de um teste de evocações livres (Wachelke \& Wolter, 2011)

\section{Resultados e Discussão}

\section{Medida de abuso digital nos relacionamentos amorosos}

Considerando a medida utilizada que mensura o envolvimento no ADRA em algum momento da vida, os dados revelaram índices de 87,6\% (média de 2,41) para vítimas de Controle/Monitoramento, 92,6\% (média de 2,36), perpetradores de Controle/Monitoramento; 34,7\% (média de 1,25), vítimas de Agressão Direta, e 25,1\% (média de 1,14), perpetradores de Agressão Direta. Tais achados apontam para uma alta prevalência de envolvimento no ADRA que pode ser explicada pelo uso indiscriminado das tecnologias de comunição socialmente, bem como, pela sua inserção dentro das relações íntimas.

Resultados similares são encontrados na literatura, os quais indicam ser o ADRA um comportamento cotidiano entre casais com índices expressivos, destacando o controle/ monitoramento como o tipo de abuso digital entre parceiros íntimos mais frequente (Borrajo et al., 2015a; Van Ouytsel, Ponnet, Walrave \& Temple, 2017; Zweig et al., 2013).

\section{Análise Prototípica}

A partir da questão de evocação livre com o estímulo indutor "abuso digital nos relacionamentos amorosos", obtiveram-se 2.231 evocações com 649 palavras diferentes. A frequência média das evocações foi de 33,22 e a ordem média de evocação (OME) foi de 2,75. A frequência mínima considerada para inclusão das palavras nos quadrantes foi de 15 , pouco mais de 3\% do tamanho da amostra. Com base nesses valores, foi gerado um diagrama com quatro quadrantes, conforme verificado na Tabela 1. Os resultados apresentam as estruturas representacionais do grupo de pertença estudado, universitários, acerca do abuso digital nos relacionamentos amorosos. 
Tabela 1

Estrutura das Representações Sociais do Abuso digital nos relacionamentos amorosos (Frequência mínima de 15).

\begin{tabular}{|c|c|c|c|c|c|c|}
\hline \multicolumn{4}{|c|}{$\mathrm{OME}<=>2,75$} & \multicolumn{3}{|c|}{$\mathrm{OME}<=>2,75$} \\
\hline \multirow{2}{*}{$\begin{array}{l}\text { Frequência } \\
\text { Média }\end{array}$} & \multicolumn{3}{|c|}{ Núcleo Central } & \multicolumn{3}{|c|}{ Periferia Primária } \\
\hline & Evocações & $f$ & OME & Evocações & $F$ & OME \\
\hline \multirow{6}{*}{$>33,21$} & Ciúme & 102 & 2.6 & Falta de respeito & 64 & 2.8 \\
\hline & Controle & 47 & 2.6 & Exposição & 54 & 2.8 \\
\hline & Invasão & 38 & 2.3 & Medo & 52 & 3.4 \\
\hline & Nudes & 35 & 1.9 & Desconfiança & 51 & 3.2 \\
\hline & & & & Insegurança & 46 & 3.0 \\
\hline & & & & Violência & 40 & 2.9 \\
\hline \multirow{2}{*}{$\begin{array}{l}\text { Frequência } \\
\text { Média }\end{array}$} & \multicolumn{3}{|c|}{ Zona de Contraste } & \multicolumn{3}{|c|}{ Periferia Secundária } \\
\hline & Evocações & $f$ & $\mathrm{OME}$ & Evocações & $F$ & OME \\
\hline \multirow{10}{*}{$<33,21$} & Facebook & 32 & 2.1 & Posse & 33 & 3.1 \\
\hline & Privacidade & 28 & 2.7 & Traição & 30 & 2.9 \\
\hline & Senha & 25 & 2.0 & Machismo & 27 & 3.0 \\
\hline & Fotos & 25 & 2.0 & Raiva & 23 & 3.0 \\
\hline & Perseguição & 24 & 2.4 & Abuso & 22 & 3.0 \\
\hline & Stalkear & 16 & 2.2 & Ameaça & 18 & 3.1 \\
\hline & Whatsapp & 16 & 2.6 & Tristeza & 17 & 3.6 \\
\hline & Vigilância & 15 & 2.3 & Vergonha & 17 & 3.3 \\
\hline & & & & Crime & 17 & 3.2 \\
\hline & & & & Agressão & 18 & 2.9 \\
\hline
\end{tabular}

Conforme observado, à esquerda, no quadrante superior, denominado de núcleo central, são apresentadas as palavras de alta frequência (maior que a média) e baixa ordem média de evocação, ou seja, que foram mais prontamente evocadas. Os elementos representacionais desse quadrante são responsáveis, segundo Abric (1998) por criarem ou transformarem os significados dos quadrantes periféricos. Além disso, apresentam um caráter mais estável, permanente, consensual, relacionando-se a histórica coletiva do grupo de pertença.

Neste eixo, o ciúme representa a espinha dorsal das representações sociais do ADRA, o qual mobiliza ações abusivas (controle, invasão e envio de nudes). Tal elemento carrega consigo um conjunto de significados tecidos pelo homem ao longo da história da espécie, assim como aponta Spink (1993). Neste sentido, verifica-se que o ciúme desempenha um papel preponderante nas relações românticas historicamente, apresentando-se como um conceito atemporal. Por exemplo, no período romano, funcionava como um protetor da "moral do homem"; no renascimento, como uma atitude frente a ameaças à fidelidade conjugal; na modernidade, como um temor pela perca do objeto de amor. Finalmente, na contemporaneidade, como um resgate da sensação de segurança e confiança (Almeida \& Lourenço, 2011; Baroncelli, 2011).

Por atravessar diferentes épocas da história do ser humano, algumas manifestações de ciúmes podem ser consideradas familiares, percebidas como opositoras da apatia; valorizadas socialmente; ou ainda, tratadas como sinônimo de amor, não obstante, podem mascarar ações abusivas (Almeida \& Lourenço, 2011; Baroncelli, 2011; Wright, 2017). Acerca disso, uma evidência bastante recorrente empiricamente é a de que o ciúme pode se apresentar como um importante gatilho para atos violentos dentro dos relacionamentos amorosos; ou ainda, a sua manifestação pode vim acompanhada de ameaças, agressões físicas e verbais (Daspe, 
Vaillancourt-Morel, Lussier, \& Sabourin, 2018; Kaufman-Parks, Longmore, Giordano, \& Manning, 2018; Wright, 2017).

Com o auxílio dos dispositivos tecnológicos, o ciúme pode ser piorado, de modo que assim como no cotidiano real, os parceiros íntimos utilizam as TICS para expressar o ciúme através de ações abusivas como: compartilhamento de fotos íntimas sem consentimento; invasão de privacidade, comportamentos de investigação (stalkear), posse, e perseguição.

Tais achados corroboram pesquisas anteriores (Borrajo et al., 2015a; Deans \& Bhogal, 2019), bem como, estão coerente com o que Christofides, Muise, e Desmarais (2009) destaca ao mencionar que o ciúme, embora atuar como um alívio a curto prazo da insegurança relacional, pode favorecer ao aparecimento de mais episódios de ciúme a médio e a longo prazo.

Ainda sobre os elementos emergidos no núcleo central estão ancorados na incerteza relacional contemporânea, bem como, na concepção de dominância dentro dos relacionamentos íntimos, fazendo com que indivíduos, em prol da manutenção das suas relações, emitam comportamentos virtuais abusivos e de ciúme, como: controlar, invadir, disseminar fotos íntimas sem consentimento (nudes).

Acerca disso, Bauman (2004) já mencionava que a cultura das relações íntimas atuais, embora se baseiem em pilares que promovam a autonomia, que se distanciam do tipo de intimidade amorosa engessado socialmente, antagonicamente, em razão da liquidez do compromisso, e com a consequente descartabilidade das relações, reproduzem vínculos mais inseguros. Frente a essa ambiguidade, tal relação necessitará de vigilância e defesa para que se sustente, sendo expressado pelo ciúme, intensa vigília, monitoramento e controle.

Por outro lado, o ADRA se pauta na lógica de dominação e assimetria de poder entre parceiros íntimos, em que para exercer domínio sobre o outro, o individuo busca controlá-lo, de modo que o abuso seria a expressão máxima do domínio. A despeito disso, evocando as ideias de Foucault sobre relações de poder que permeiam toda sociedade, Fernandes (2010) destaca que nos relacionamentos íntimos o poder pode ser exercido sob influência da cultura, do gênero, ou de papéis sociais impostos pelo sistema patriarcal.

Chama atenção ainda, para o fato de que os elementos mais proeminentes no núcleo central: "ciúme" e "controle" são dimensões estudadas em distintas pesquisas acerca do ADRA (Borrajo et al. 2015a; Reed et al., 2017; Sánchez et al., 2015), sendo conceitos imprescindíveis para a definição desse fenômeno. Neste sentido, esse achado aponta para uma convergência do conhecimento socialmente elaborado com o universo retificado.

Em última análise, destaca-se o elemento "nudes", que no contexto de violência é um termo que faz alusão à conteúdo íntimo utilizado pelo agressor para denegrir, difamar, chantagear a vítima, ou até mesmo obrigá-la a ter relações sexuais com ele (estupro virtual). Tais ações apontam para um fenômeno social contemporâneo, denominado "porn revenge" ou "pornografia de vingança, o qual vem sendo indicado, na literatura, como intimamente ligado a violência de gênero, uma vez que a maioria das vítimas são mulheres (Bates, 2017; Citron \& Franks, 2014; McGlynn, Rackley, \& Houghton, 2017).

Quanto às representações dos sistemas periféricos (segundo, terceiro e quarto quadrante), de acordo com Abric (1998), essas apresentam um caráter mais flexível, sendo sensíveis ás circunstâncias, integrando vivências individuais.

O segundo quadrante, denominado sistema periférico próximo, foi composto por palavras com alta frequência (maior que a média) e alta ordem de evocação; em outras palavras, aquelas que não foram tão prontamente evocadas. Esse sistema mantém o núcleo central e integra a estas novas informações, como: "falta de respeito", "exposição", "violência", "desconfiança", "insegurança", e "medo". Tais elementos estão ancorados violação dos direitos de privacidade e nos sentimentos negativos ocasionados por ela, apontando o ADRA como uma forma de violar, desrespeitar e expor a vida íntima do parceiro(a), causando-lhe insegurança e medo. 
Conforme se verifica, assim como na violência presencial entre parceiros íntimos (Barros et al., 2016; Zerubavel, Messman-Moore, DiLillo, \& Gratz, 2018), o medo e a insegurança são impostos às vítimas de ADRA. Tais elementos são preocupantes uma vez que podem ser responsáveis pela emergência de sofrimento relacional refletidos em danos psicológicos e de insatisfação conjugal. Além disso, alertam para as sensações de ameaça e vulnerabilidade frente aos perigos conferidos pela tecnologia. De modo que, no espaço virtual, essa insegurança pode ser mais agravante pelo fato de ainda ser inicial o debate sobre políticas de enfrentamento da intimidação virtual.

No quadrante inferior esquerdo, chamado de zona de contraste, encontram-se as palavras com uma menor frequência (abaixo da média) com baixa ordem de evocação, ou seja, prontamente evocados. Nesse agrupamento o ADRA foi associado ao "facebook" e "whatsap" remetendo aos espaços de ocorrência dos abusos digitais nos relacionamentos amorosos, indicado pelas redes sociais.

As redes sociais, enquanto ambientes propícios para prática do ADRA, se justifica pela sua facilidade de acesso e suas múltiplas fontes de informação, como: acompanhamento das últimas postagens, fotos ou interações com outras pessoas. Sendo assim, o conhecimento da senha desses dispositivos, seja por roubo ou consentimento, aumenta ainda mais as possibilidades de vigilância.

Em alguns casos, tendo em vista a natureza pública das redes sociais, este abuso pode ultrapassar a esfera íntima do casal, assumindo proporções mais amplas através de ações que envolvem compartilhamento ilimitado de fotos ou vídeos íntimos do parceiro sem consentimento, ou ainda postagens humilhantes acerca dele publicamente. Esses dados se confirmam nos estudos de Van Ouytsel et al. (2016), os quais encontraram que o comportamento de monitoramento tem sido associado à quantidade de redes sociais.

Quanto as evocações: fotos, privacidade, senhas, vigilância e perseguição, apontam para as formas de ocorrência do ADRA na sociabilidade digital, em que atos como: inspecionar, acompanhar constantemente, perseguir, invadir a privacidade, publicar fotos íntimas sem consentimento, são possibilitados pelo acesso à internet. Tais ações se coadunam com os comportamentos do ADRA elencados em estudos anteriores (Borrajo et al., 2015a; Flach \& Deslanches, 2017; Van Ouytsel et al., 2016).

Por fim, no quarto e último quadrante (inferior direito) indicam os elementos de menor frequência (abaixo da média) e maior ordem de evocação, a saber: posse, traição, machismo, raiva, abuso, ameaça, tristeza, vergonha, crime, agressão. Nesse eixo permitiram-se variações pessoais, em que predominaram-se aspectos conceituais do ADRA, bem como, suas causas e consequências, os quais perpassam elementos psicoafetivos e sociais.

No que tange aos aspectos conceituais, verifica-se que o ADRA é definido de maneira mais ampla enquanto um tipo de agressão e abuso, bem como de maneira específica através da ameaça, enquanto estratégia para chantagear o outro. O ADRA foi descrito ainda como sinônimo de crime, indicando ser um problema que transgride direitos, liberdades e garantias. Esse elemento faz alusão ao conhecimento de iniciativas jurídicas de combate ao delito virtual, como é o caso da Lei $13.718 / 2018$ sobre crimes sexuais.

Ainda acerca do quarto quadrante, chama atenção para o fato de que mesmo o presente estudo utilizar um "estímulo indutor" que carregue consigo o termo "abuso", tal elemento, e correlatos, são evocados em menor frequência. O que pode indicar uma falta de percepção sobre o sentido real do ADRA e suas motivações, seja em virtude de uma visão distorcida do fenômeno, em que ações abusivas são percebidas como amor ou brincadeira (Borrajo, Gámez-Guadix, \& Calvete, 2015b; Muñoz-Rivas, Gámez-Guadix, Graña, \& Fernández, 2010).

No que tange aos elementos causais, o elemento "machismo" faz referência a concepção de supremacia masculina, em que ao homem é imposto dominar, agredir, invadir, tornando tais comportamentos aceitáveis e naturalizados também no contexto virtual. Além disso, o ADRA pode ser ocasionado pela ameaça, mesmo que remota, de traição do parceiro, ou perca da figura de 
posse, uma vez que a tecnologia possibilita uma infinidade de novas interações sociais. Finalmente aponta para raiva como imperativo ao ADRA, aspecto destacado por pesquisas na área tanto ara mobilizar comortamentos agressivos presenciais ou virtuais (Cavalcanti \& Pimentel, 2016; Deans \& Bhogal, 2019; Watkins, Maldonado, \& DiLillo, 2016).

Quanto aos aspectos que remetem as consequências do ADRA são descritas a vergonha $\mathrm{e}$ tristeza, elementos esses que quando alterados podem levar a estados de humor preocupantes, como: depressão, ansiedade, podendo levar ao suicidio. Tais implicações do ADRA são descritas no estudo de revisão de Flach e Deslandes (2017) assim como também: distúrbios no sono, envolvimento em comportamentos delinquentes, e implicações negativas para o rendimento escolar.

\section{Considerações finais}

A partir dos dados coletados foi possível constatar uma alta prevalência de envolvimento no ADRA, acima de $20 \%$ para Agressão Direta, e $80 \%$ para o controle e monitoramento, o que aponta para necessidade de novas investigações no Brasil. Além disso, chama atenção para necessidade de intervenções com vistas a minimizar esse fenômeno, uma vez que são apontadas graves consequências aos seus envolvidos.

No que tange as Representações Sociais, o núcleo central do ADRA foi ancorado na incerteza relacional contemporânea e na concepção de dominância dentro dos relacionamentos íntimos. Quanto as evocações do núcleo periférico o ADRA foi associado à violação dos direitos à privacidade, remetendo as redes sociais como os principais espaços de ocorrência para ações de vigilância, perseguição, invasão e publicação de fotos íntimas sem consentimento. Além disso, foi representado como um tipo de agressão que perpassa causas e implicações psicoafetivas e sociais.

Os resultados revelaram ainda que o "ciúme" desempenha um papel preponderante no ADRA, uma vez que aparece como elemento central e organizador das RS desse fenômeno, mobilizando ações virtuais abusivas como: compartilhamento de fotos íntimas sem consentimento; invasão de privacidade, comportamentos de investigação (stalkear) e de posse, perseguição.

Tais achados sugerem que estudar o ADRA sob o enfoque das RS se faz pertinente, tendo em vista que esse fenômeno apresenta carcaterísticas complexas, abrangendo questões de ordens individuais, sociais, culturais e históricas. Por essa razão, intervenções direcionadas a combater esse fenômeno deve tomar por base uma visão poliocular.

Embora resultados relevantes, a presente pesquisa não está isenta de limitações, como por exemplo a utilização de uma amostra não probabilística, a qual não se pode pressupor a generalização dos dados. Além disso, observa-se um desequilíbrio entre os sexos na amostra, de modo que as mulheres representam expressivamente a maior parte da pesquisa, o que pode ter enviesado, por exemplo no aparecimento da palavra "machismo".

Neste sentido, entende-se que novos estudos seriam igualmente relevantes, tais como o desenvolvimento de pesquisas longitudinais que analisem como as representações acerca do fenômeno ADRA se modificam ao longo da idade, bem como, investigações transculturais que busquem conhecer as RS em outras culturas. Sugerem-se ainda estudos que permitam explorar melhor a relação entre representação social e comportamento, demonstrando se representações, crenças e atitudes podem predispor comportamentos de abuso online entre parceiros, ou ainda, de violência presencial. 


\section{Referências}

Abric, J. C. (1998). A abordagem estrutural das representações sociais. Estudos interdisciplinares de representação social, 2(1998), 27-38.

Almeida, T., \& Lourenço, M. L. (2011). Ciúme romântico: Um breve histórico, perspectivas, concepções correlatadas e seus desdobramentos para os relacionamentos amorosos. Revista de Psicologia, 2(2), 18-32.

Baroncelli, L. (2011). Amor e ciúme na contemporaneidade: reflexões psicossociológicas. Psicologia \& Sociedade, 23(1), 163-170. doi: 10.1590/S0102-71822011000100018.

Barros, E. N., Silva, M. A., Falbo Neto, G. H., Lucena, S. G., Ponzo, L., \& Pimentel, A. P. (2016). Prevalência e fatores associados à violência por parceiro íntimo entre mulheres em Recife/Pernambuco, Brasil. Ciência \& Saúde Coletiva, 21(2), 591-598. doi: 10.1590/141381232015212.10672015

Bates, S. (2017). Revenge porn and mental health: A qualitative analysis of the mental health effects of revenge porn on female survivors. Feminist Criminology, 12(1), 22-42. doi: $10.1177 / 1557085116654565$

Bauman, Z. (2004). Amor líquido: sobre a fragilidade dos laços humanos. Zahar.

Beserra, M. A., Leitão, M. N. C., Fernandes, M. I. D., Scatena, L., Vidinha, T. S. S., Silva, L. M. P., \& Ferriane, M. G. C. (2015). Prevalência de Violência no Namoro entre Adolescentes de Escolas Públicas de Recife/Pe: Brasil. Revista de Enfermagem Referência, serIV(7), 9199. doi: 10.12707/RIV15006

Borrajo, E., Gámez-Guadix, M., Pereda, N., \& Calvete, E. (2015a). The development and validation of the cyber dating abuse questionnaire among young couples. Computers in human behavior, 48, 358-365. doi: 10.1016/j.chb.2015.01.063

Borrajo, E., Gámez Guadix, M., \& Calvete, E. (2015b). Justification beliefs of violence, myths about love and cyber dating abuse. Psicothema, 27(4), 327-333. doi: 10.7334/psicothema2015.59.

Brownridge, D. A., Taillieu, T., Chan, K. L., \& Piotrowski, C. (2018). Understanding the Elevated Prevalence of Intimate Partner Violence in Interracial Relationships. Journal of interpersonal violence, 0886260518781803 , doi: 10.1177/0886260518781803

Burke, S. C., Wallen, M., Vail-Smith, K., \& Knox, D. (2011). Using technology to control intimate partners: An exploratory study of college undergraduates. Computers in Human Behavior, 27, 1162-1167. doi: 10.1016/j.chb.2010.12.010

Cavalcanti, J. G., \& Pimentel, C. E. (2016). Personality and aggression: A contribution of the General Aggression Model. Estudos de Psicologia (Campinas), 33(3), 443-451. doi: 10.1590/1982-02752016000300008

Cavalcanti, J. G., Coutinho, M. D. P. D. L., Nascimento, A. M. D., \& Pinto, A. V. D. L. (2020). Psychometric Properties of the Cyber Dating Abuse Questionnaire. Psico-USF, 25(2), 285296. doi: 10.1590/1413-82712020250207

Christofides, E., Muise, A., \& Desmarais, S. (2009). More information than you ever wanted: Does facebook bring out the green-eyed monster of jealousy? Cyber Psychology \& Behavior, 12(4), 441-444. doi: 10.1089/cpb.2008.0263

Citron, D. K., \& Franks, M. A. (2014). Criminalizing revenge porn. Wake Forest L. Rev., 49, 345.

Conceição, T. B., Bolsoni, C. C., Lindner, S. R., \& Coelho, E. B. S. (2018). Assimetria e simetria de gênero na violência por parceiro íntimo em pesquisas realizadas no Brasil. Ciência \& Saúde Coletiva, 23, 3597-3607. doi: 10.1590/1413-812320182311.23902016.

Cutbush, S., Ashley, O., Kan, M., \& Hall, D. (2010). Electronic aggression among adolescent dating partners: Demographic correlates and associations with academic performance and other types of violence. Poster presented at the American Public Health Association, 
annual meeting, November 6-10, Denver, CO. Retrieved from: https://apha.confex.com/apha/138am/webprogram/Paper229575.html

Daspe, M. Ė., Vaillancourt-Morel, M. P., Lussier, Y., Sabourin, S. (2018). Facebook Use, Facebook Jealousy, and Intimate Partner Violence Perpetration. Cyberpsychol Behav Soc Netw, 21(9), 549-555. doi: 10.1089/cyber.2018.0159

Deans, H., \& Bhogal, M. S. (2019). Perpetrating cyber dating abuse: A brief report on the role of aggression, romantic jealousy and gender. Current Psychology: A Journal for Diverse Perspectives on Diverse Psychological Issues, 38(5), 1077-1082. doi: 10.1007/s12144017-9715-4

Durán, M., \& Martínez-Pecino, R. (2015). Ciberacoso mediante teléfono móvil e Internet en las relaciones de noviazgo entre jóvenes. Comunicar, 22(44), 159-167. doi: 10.3916/C442015-17

Elísio, R., Neves, S., \& Paulos, R. (2018). A violência no namoro em casais do mesmo sexo: discursos de homens gays. Revista Crítica de Ciências Sociais, 117, 47-72. doi: $10.4000 /$ rccs. 8149

Fernandes, A. R. (2010). O poder nas relações conjugais: uma investigação fenomenológica sobre as relações de poder no casamento. São Paulo: Annablume.

Flach, R. M. D., \& Deslandes, S. F. (2017). Abuso digital nos relacionamentos afetivo-sexuais: uma análise bibliográfica. Cadernos de Saúde Pública, 33(7), e00138516. doi: 10.1590/0102-311X00138516.

Hancock, K., Keast, H., \& Ellis, W. (2017). The impact of cyber dating abuse on self-esteem: The mediating role of emotional distress. Cyberpsychology: Journal of Psychosocial Research on Cyberspace, 11(2). doi: 10.5817/CP2017-2-2

Jodelet, D., \& Moscovici, S. (1989). Folies et représentations sociales. Presses universitaires de France.

Jodelet, D. (2001). Representações sociais: um domínio em expansão. Em D. Jodelet, D. (Org.), As representações sociais, (pp. 17-44). Rio de Janeiro: EdUERJ.

Kaufman-Parks, A. M., Longmore, M. A., Giordano, P. C., \& Manning, W. D. (2018). Inducing jealousy and intimate partner violence among young adults. Journal of Social and Personal Relationships, 36(9), 2802-2823. doi: 10.1177/0265407518802451

McGlynn, C., Rackley, E., \& Houghton, R. (2017). Beyond 'Revenge Porn': The continuum of image-based sexual abuse. Feminist Legal Studies, 25(1), 25-46. doi: 10.1007/s10691-0179343-2

Melander, L. A. (2010). College students' perceptions of intimate partner cyber harassment. Cyberpsychology, behavior, and social networking, 13(3), 263-268. doi: 10.1089=cyber.2009.0221.

Moscovici, S. (1981). La psychanalyse, son image et son public. Paris: Press Universitaires de France.

Moscovici, S. (2012). A Psicanálise, sua Imagem e seu Público. Petrópolis: Vozes

Muñoz-Rivas, M. J., Gámez-Guadix, M., Graña, J. L., \& Fernández, L. (2010). Violencia en el noviazgo y consumo de alcohol y drogas ilegales entre jóvenes españoles. Adicciones (Palma de Mallorca), 22, 125-134. doi: 10.20882/adicciones.201

Neves, S. (2008). Amor, poder e violências na intimidade. Coimbra: Quarteto.

Peskin, M. F., Markham, C. M., Shegog, R., Temple, J. R., Baumler, E. R., Addy, R. C., ... \& Emery, S. T. (2017). Prevalence and correlates of the perpetration of cyber dating abuse among early adolescents. Journal of youth and adolescence, 46(2), 358-375. doi: 10.1007/s10964-016-0568-1

Pimentel, C. E., Moura, G. B. D., \& Cavalcanti, J. G. (2017). Acceptance of Dating Violence Scale: Checking its psychometric properties. Psico-USF, 22(1), 147-159. doi: 10.1590/1413-82712017220113 
Ramos, M. C., Miller, K. F., Moss, I. K., \& Margolin, G. (2017). Perspective-Taking and Empathy Mitigate Family-of-Origin Risk for Electronic Aggression Perpetration Toward Dating Partners: A Brief Report. Journal of interpersonal violence, 0886260517747605. doi: $10.1177 / 0886260517747605$

Reed, L. A., Tolman, R. M., \& Ward, L. M. (2017). Gender matters: Experiences and consequences of digital dating abuse victimization in adolescent dating relationships. Journal of adolescence, 59, 79-89. doi: 10.1016/j.adolescence.2017.05.015

Runions, K., Shapka, J. D., Dooley, J., \& Modecki, K. (2013). Cyber-aggression and victimization and social information processing: Integrating the medium and the message. Psychology of violence, 3(1), 9-26. doi: 10.1037/a0030511

Sánchez, V., Muñoz-Fernández, N., \& Ortega-Ruíz, R. (2015). "Cyberdating Q_A": An instrument to assess the quality of adolescent dating relationships in social networks. Computers in human behavior, 48, 78-86. doi: 10.1016/j.chb.2015.01.006

Santos, A. M. R., \& Caridade, S. M. M. (2017). Violência nas relações íntimas entre parceiros do mesmo sexo: estudo de prevalência. Trends in Psychology, 25(3), 1341-1356. doi: $10.9788 / \mathrm{tp} 2017.3-19 \mathrm{pt}$

Slonje, R. \& Smith, P. K. (2008). Cyberbullying: Another main type of bullying? Scandinavian journal of psychology, 49(2), 147-154. doi: 10.1111/j.1467-9450.2007.00611.x

Smith, K., Cénat, J. M., Lapierre, A., Dion, J., Hébert, M., \& Côté, K. (2018). Cyber dating violence: Prevalence and correlates among high school students from small urban areas in Quebec. Journal of affective disorders, 234, 220-223. doi: 10.1016/j.jad.2018.02.043

Spink, M. J. P. (1993). O conceito de representação social na abordagem psicossocial. Cadernos de Saúde Pública, 9(3), 300-308. doi: 10.1590/S0102-311X1993000300017

Stonard, K. E., Bowen, E., Lawrence, T. R., \& Price, S. A. (2015). The relevance of technology to the nature, prevalence and impact of Adolescent Dating Violence and Abuse: A research synthesis. Aggression and Violent Behavior, 19(4), 390-417. doi: 10.1016/j.avb.2014.06.005

Tokunaga, R. S. (2011). Social networking site or social surveillance site? Understanding the use of interpersonal electronic surveillance in romantic relationships. Computers in Human Behavior, 27(2), 705-713. doi: 10.1016/j.chb.2010.08.014

Turell, S. C., Brown, M., \& Herrmann, M. (2018). Disproportionately high: an exploration of intimate partner violence prevalence rates for bisexual people. Sexual and Relationship Therapy, 33(1-2), 113-131. doi: 10.1080/14681994.2017.1347614

Vala, J. (2013). Psicologia Social (9 ed.) Lisboa: Fundação Calouste Gulbenkian.

Van Ouytsel, J., Ponnet, K., \& Walrave, M. (2016). Cyber dating abuse victimization among secondary school students from a lifestyle-routine activities theory perspective. Journal of interpersonal violence, 0886260516629390 . doi: 10.1177/0886260516629390

Van Ouytsel, J., Ponnet, K., Walrave, M., \& Temple, J. R. (2017). Adolescent cyber dating abuse victimization and its associations with substance use, and sexual behaviors. Public health, 135, 147-151. doi: 10.1016/ j.puhe.2016.02.011

Watkins, L. E., Maldonado, R. C., \& DiLillo, D. (2016). The Cyber Aggression in Relationships Scale: a new multidimensional measure of technology-based intimate partner aggression. Assessment, 25(5), 608-626. doi: 10.1177/1073191116665696

Wachelke, J., \& Wolter, R. (2011). Critérios de construção e relato da análise prototípica para representações sociais. Psicologia: Teoria e pesquisa, 27(4), 521-526. doi: 10.1590/S010237722011000400017

Wright, M. F. (2017). Intimate partner aggression and adult attachment insecurity: The mediation of jealousy and anger. Evolutionary Behavioral Sciences, 11(2), 187-198. doi: $10.1037 /$ ebs0000097 
Wu, E., El-Bassel, N., McVinney, L. D., Hess, L., Fopeano, M. V., Hwang, H. G., ... \& Mansergh, G. (2015). The association between substance use and intimate partner violence within Black male same-sex relationships. Journal of interpersonal violence, 30(5), 762-781. doi: $10.1177 / 0886260514536277$

Zerubavel, N., Messman-Moore, T. L., DiLillo, D., \& Gratz, K. L. (2018). Childhood Sexual Abuse and Fear of Abandonment Moderate the Relation of Intimate Partner Violence to Severity of Dissociation. Journal of Trauma \& Dissociation, 19(1), 9-24. doi: 10.1080/15299732.2017.1289491

Zweig, J. M., Dank, M., Yahner, J., \& Lachman, P. (2013). The rate of cyber dating abuse among teens and how it relates to other forms of teen dating violence. Journal of youth and adolescence, 42(7), 1063-1077. doi: 10.1007/s10964-013-9922-8

Participação dos autores: a) Planejamento e concepção do trabalho; b) Coleta de dados; c) Análise e interpretação de dados; d) Redação do manuscrito; e) Revisão crítica do manuscrito.

J.G.C. contribuiu em a,b,c,d,e; M.P.L.C. em b,c,d,e; A.V.L.P.em b,c,d,e.

Editora científica responsável: Dra. Cecilia Cracco 